\title{
Effects of patch characteristics and within patch heterogeneity on the accuracy of urban land cover estimates from visual interpretation
}

\author{
Weiqi Zhou $\cdot$ M. L. Cadenasso
}

Received: 5 January 2012 / Accepted: 6 July 2012

(C) Springer Science+Business Media B.V. 2012

\begin{abstract}
Landscape ecology links landscape pattern to ecological function. Achieving this goal hinges on accurate depiction and quantification of pattern, which is frequently done by visually interpreting remotely sensed imagery. Therefore, understanding both the accuracy of that interpretation and what influences its accuracy is crucial. In addition, imagery is pixel-based but landscape pattern exists, more realistically, as irregularly shaped patches. Patches may contain only one feature type such as trees, but, in some landscapes, patches may contain several different types of features such as trees and buildings. Using a patch-based approach, this paper investigates two types of variables - whole-patch and within-patchthat are hypothesized to influence the accuracy of visually estimating the cover of features within patches. A highly accurate reference map, obtained from object-based classification, was used to evaluate the accuracy of visual estimates of cover within patches. The effects of the variables on the accuracy of
\end{abstract}

W. Zhou ( $\bowtie)$

State Key Laboratory of Urban and Regional Ecology, Research Center for Eco-Environmental Sciences, Chinese Academy of Sciences, Shuangqinglu 18, Beijing 100085, China

e-mail: wzhou@rcees.ac.cn

M. L. Cadenasso

Department of Plant Sciences, University of California, Mail Stop 1, 1210 PES, One Shields Avenue, Davis, CA 95616, USA these estimates were tested using logistic regressions and multimodel inferential procedures. Though all variables significantly affected the accuracy, the within-patch configuration of features is the most significant factor. In general, errors of cover estimates are more likely to occur when patches are smaller or have more complex shapes, and features within a patch are (1) more diverse; (2) more fragmented; (3) more complex in shape; and (4) physically less connected. These results provide an important first step towards a quantitative, spatially explicit model for predicting error of cover estimates and determining under what circumstances estimation error is most likely to occur.

Keywords Visual interpretation - Object-based classification · HERCULES · Accuracy · Land cover composition and configuration · Pattern analysis . Spatial heterogeneity $\cdot$ Urban systems

\section{Introduction}

Landscape ecology focuses on understanding the reciprocal link between pattern and process (Turner et al. 2001; Wu and Hobbs 2002). Building this understanding requires accurate quantification of landscape pattern at the grain and extent appropriate for a specific research question (Gustafson 1998; Turner 2005; Shao and $\mathrm{Wu}$ 2008). Quantifying 
landscape pattern primarily relies on thematic maps, and these maps are frequently derived from visual interpretation of remotely sensed imagery (Turner et al. 2001; Groom et al. 2006; Iverson 2007). Therefore, pattern quantification, and consequently our ability to apply it to understanding and predicting variation in ecological processes in the landscape, hinges on accurate classification of remotely sensed imagery (Wu and Hobbs 2002; Iverson 2007; Li and $\mathrm{Wu}$ 2007; Shao and $\mathrm{Wu}$ 2008). Because this is fundamental to advancing research in landscape ecology, it is crucial that we assess what influences the accuracy of these classifications.

Visual interpretation of patterns and land cover features is an approach used both by landscape ecologists and remote sensing specialists. Visual interpretation, frequently referred to as photo-interpretation or manual interpretation, is the process by which human analysts extract information by visually inspecting an image (Lillesand and Kiefer 2004; Richards and Jia 2006). Within the remote sensing literature, visual interpretation has typically been used for digitizing individual landscape features and ground truthing images (Lillesand and Kiefer 2004; Richards and Jia 2006). In landscape ecology, visual interpretation is a well-established method for patch mapping and classification (e.g., Fensham et al. 2002; Allard et al. 2003; Cadenasso et al. 2007; Gill et al. 2008; Heiskanen et al. 2008; Ståhl et al. 2011).

Patches in the landscape can be mapped based on many different criteria such as variation in plant community composition or land use/land cover. Patches can also be delineated based on a contrast in an ecological process, such as rates of denitrification. The appropriate criteria used to delineate patches depend on the specific research question being addressed (Cadenasso et al. 2003). A patch is a spatial unit that has a specific location and dimensionality. Therefore, patches can be quantitatively described by size and shape as well as location relative to other patches (Gustafson 1998). In addition, patches may contain more than one feature. For example, patches delineated using the criteria of land use may all be residential but these patches contain types and amounts of features such as buildings, impervious surfaces, and trees (e.g., Cadenasso et al. 2007; Gill et al. 2008).

Patch mapping and classification commonly consists of two steps: (1) delineating patch boundaries, and (2) deriving attribute values of with-patch features such as percent cover of trees and impervious surfaces (e.g., Allard et al. 2003; Cadenasso et al. 2007; Gill et al. 2008). The variation in delineating boundaries by different interpreters or the same interpreter over time has been extensively assessed (Congalton and Mead 1983; Cherrill and McClean 1995; Ellis et al. 2006). This paper, however, focuses on the accuracy of the second step-visually estimating the cover of features within the patch.

The accuracy of estimating the cover of features within a patch may be affected by the photoscales, land cover types, landscape structure, and the skills of the photo-interpreters (Fensham et al. 2002; Paine and Kiser 2003; Fensham and Fairfax 2007; Zhou et al. 2010). This accuracy has been commonly assessed and calibrated by field measurements (e.g., Fensham and Fairfax 2003; Clehmann et al. 2009) or reference data derived from other approaches (e.g., Zhou et al. 2010). Few studies, however, have quantitatively examined factors that may affect this accuracy (Fensham et al. 2002; Paine and Kiser 2003; Fensham and Fairfax 2007), and we are not aware of any that have investigated how whole-patch characteristics and within-patch characteristics affect the accuracy of visually estimating within-patch cover. We suggest that this accuracy may be influenced by whole-patch characteristics, such as the size and shape of the patch, and within-patch characteristics, such as the composition and configuration of the features (Zhou et al. 2010). Quantitatively understanding the relationship between accuracy of cover estimates and whole-patch and within-patch characteristics can provide insight into potential causes of classification errors and identify under what circumstances classification errors are most likely to occur. In addition, analyses of these relationships provide a potential tool to predict how the magnitude of error is spatially distributed in the landscape. These insights are not available from standard accuracy assessment procedures (Shao et al. 2001; Smith et al. 2002, 2003; Ellis and Wang 2006; Shao and $\mathrm{Wu} 2008)$. This paper aims to fill this gap.

The overarching goal of this study, therefore, is to determine whether patch characteristics at two scales-whole-patch and within-patch-influence the accuracy of estimating the relative cover of land cover features inside patches. Specifically, the objectives are to (1) investigate whether whole-patch characteristics, such as patch size and shape, and within-patch characteristics, such the composition and 
configuration of features, are useful predictors of errors in estimating the cover features within a patch, and (2) examine the relative strength of whole-patch and within-patch characteristics as predictors of errors in estimating the cover of features within a patch.

\section{Methods}

Study area

We have delineated and classified land cover patches in the Gwynns Falls watershed of Baltimore, MD, USA as part of the Baltimore Ecosystem Study, a Long Term Ecological Research Program funded by the National Science Foundation (www.beslter.org). The watershed is approximately $17,150 \mathrm{ha}$, and traverses an urban-suburban-rural gradient from downtown Baltimore City, through suburban and suburbanizing areas, out to the rural/suburban fringe (Fig. 1). Because of this size and range of land cover, the land cover data layer consists of patches that vary in size and shape and that vary in the relative cover of features within them. Therefore, this data layer is well suited for the goals of this research.
Data

Creating a patch layer to assess classification accuracy

A base patch layer was created using the high ecological resolution classification for urban landscapes and environmental systems (HERCULES) land cover classification. This classification was specifically developed for urban landscapes (Cadenasso et al. 2007), and focuses on the biophysical structure, specifically: (1) coarse-textured vegetation-trees and shrubs (CV), (2) fine-textured vegetation-herbs and grasses (FV), (3) bare soil, (4) pavement, (5) buildings, and (6) building typology (Cadenasso et al. 2007). Because the last feature is qualitative, it will not be discussed further. The first five features are allowed to vary independently of each other and a shift in the amount or distribution of one or more features will result in a different patch. The utility of this approach for linking landscape pattern to ecological processes is best illustrated by an example. Using standard land use/land cover classifications, residential areas in the urban landscape would be classified the same and be delineated as one continuous patch. But residential
Fig. 1 The Gwynns Falls watershed includes portions of Baltimore City and Baltimore County, MD, USA, and drains into the Chesapeake Bay

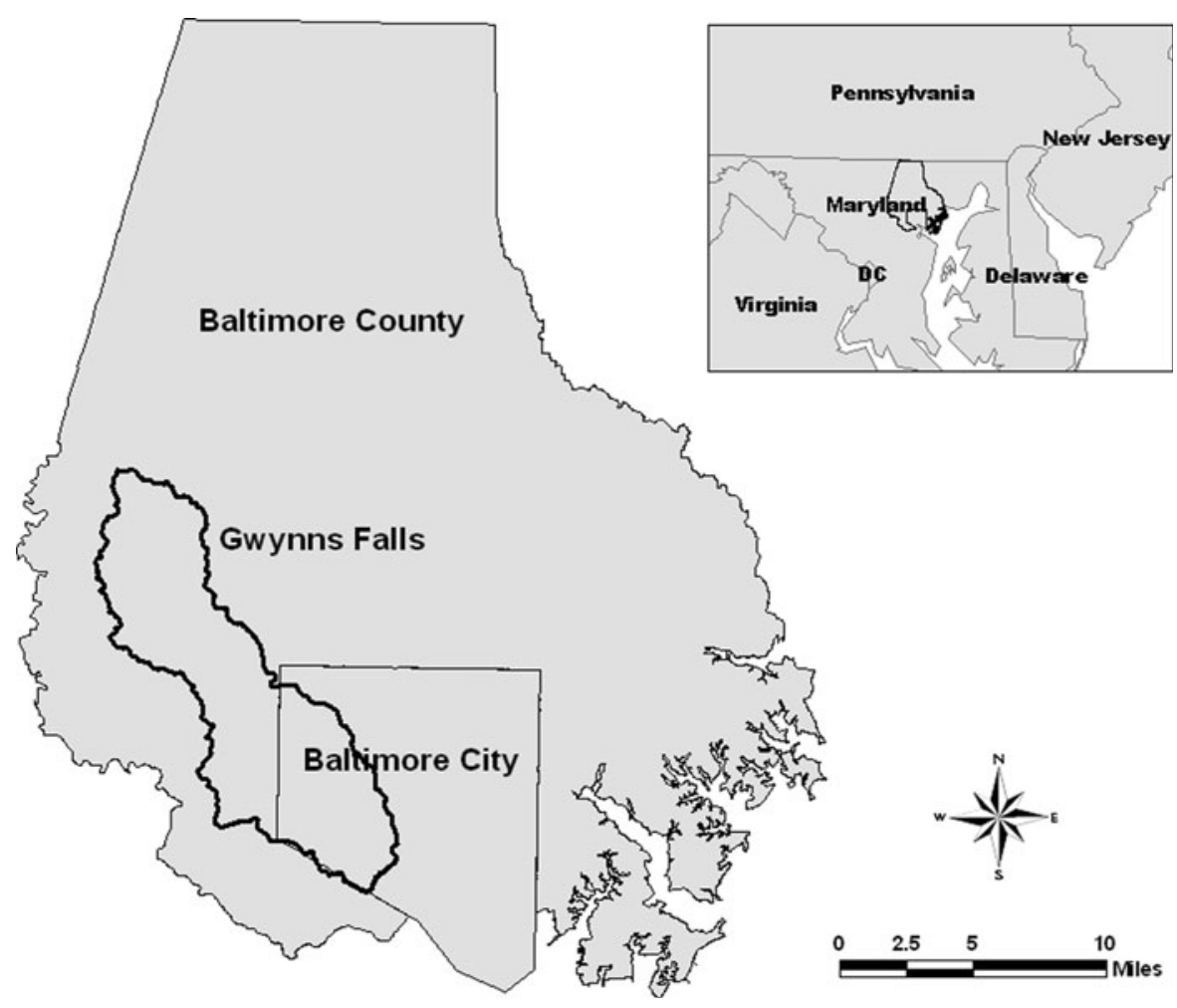



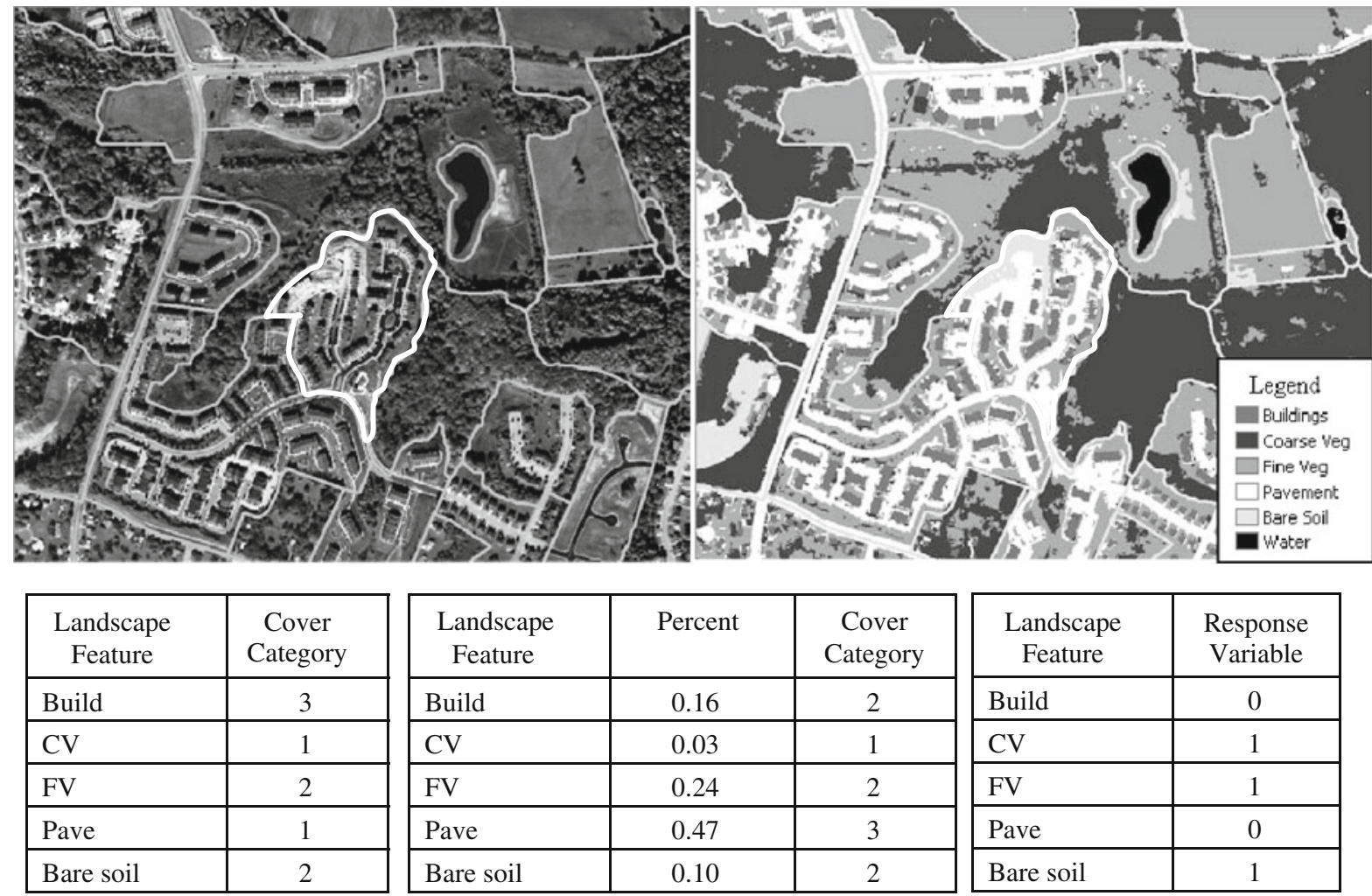

Fig. 2 HERCULES patches and within-patch cover of each of the five land cover features. Left panel high-resolution image with HERCULES patch boundaries superimposed. Right panel land cover classification map with HERCULES patch boundaries superimposed. Left table cover categories of the five features estimated by visual interpretation for the patch highlighted in the right panel. Center table: percent cover of

blocks can vary considerably in terms of building density or the presence and abundance of trees. This variation may influence ecological processes such as biodiversity or carbon storage. The HERCULES classification captures this heterogeneity in biophysical features and delineates different patches where a shift in tree abundance occurs. Patch boundaries, where possible, are digitized down the middle of a road (Fig. 2).

HERCULES patches were digitized on-screen using high-spatial resolution aerial imagery in ArcGIS $^{\text {TM }}$ (version 9.2). The imagery was collected in October 1999, has a pixel size of $0.6 \mathrm{~m}$, and is 3-band color-infrared (green: $510-600 \mathrm{~nm}$, red: $600-700 \mathrm{~nm}$, and near-infrared: $800-900 \mathrm{~nm}$ ). The imagery was orthorectified and meets the National Mapping Accuracy Standards for scale mapping of 1:3,000 (3-m accuracy with $90 \%$ confidence). A total of 2,250 the features obtained for the same highlighted patch by object based classification and the equivalent cover category. Right table: binary response variables used to represent whether the cover was correctly estimated by visual interpretation. Value of one indicates a correct categorization and zero indicates an incorrect categorization

patches were delineated in the watershed, with a mean size of $7.6 \mathrm{ha}$, and a density of approximately $13 / \mathrm{km}^{2}$.

Each HERCULES patch, most likely, contains multiple land cover features such as buildings and trees (Fig. 2). We evaluate whether the accuracy of visually estimating the cover of those features within a patch is influenced by whole-patch characteristicssize and shape - and within-patch characteristicscomposition and configuration of the features.

\section{Creating the test and reference datasets}

Using the HERCULES patch layer for the watershed, the cover of all five features within a patch were estimated using two approaches: (1) visual interpretation, and (2) object-based classification. Through visual interpretation the cover of each feature within a patch was assigned to a cover category: (0) absent, (1) 
Table 1 Summary of the accuracies for the reference land cover map obtained from object-based image analysis

\begin{tabular}{llllll}
\hline & Building & CV & FV & Pavement & $\begin{array}{l}\text { Bare } \\
\text { soil }\end{array}$ \\
\hline $\begin{array}{c}\text { User's } \\
\text { accuracy } \\
(\%)\end{array}$ & 83.6 & 97.7 & 94.9 & 91.9 & 90.0 \\
$\begin{array}{c}\text { Producer's } \\
\text { accuracy } \\
(\%)\end{array}$ & 94.4 & 94.4 & 89.3 & 88.3 & 100 \\
$\begin{array}{c}\text { Overall } \\
\text { accuracy }\end{array}$ & $92.3 \%$ & & $\begin{array}{c}\text { Kappa } \\
\text { statistic }\end{array}$ & 0.899 & \\
\hline
\end{tabular}

present to $10 \%$, (2) $11-35 \%$, (3) $36-75 \%$, and (4) $>75 \%$ (Fig. 2; Cadenasso et al. 2007). This was the test dataset. The object-based classification approach used the software eCognition (Zhou and Troy 2008) to generate the reference dataset containing the cover of features in the patches as a continuous value. The continuous cover values were then assigned into the cover categories described above (Fig. 2). The continuous values were converted to categories so that the two approaches could be compared. The overall accuracy of the object-based classification was $92.3 \%$, with producer's accuracies ranging from 88.3 to $100 \%$, and user's accuracies from 83.6 to $97.7 \%$ (Table 1).

The test and reference datasets were compared to assess the accuracy of estimating cover of features within patches based on visual interpretation. Each land cover feature was assigned a one or a zero to indicate whether or not the proportion cover estimates from visual interpretation were in the same category as those generated from the object-based approach. The resulting binary variables were used as response variables in the later logistical regressions.

\section{Whole-patch and within-patch characteristics}

Many metrics have been developed to characterize and measure spatial pattern in landscapes (Gustafson 1998; McGarigal et al. 2002). We selected commonly used metrics to describe whole-patch characteristics and the composition and configuration of features within a patch (Fig. 3; Table 2). Whole-patch metrics included: total area, total edge (i.e., perimeter), fractal dimension index, shape index, and perimeter-area ratio. Within-patch metrics included those to describe the composition of the features-percent cover of each feature and the Simpson's diversity index-and those to describe the configuration of features-area, edge, density, shape, connectivity, and proximity of each feature type (Table 2) (Gustafson 1998; McGarigal et al. 2002). Simpson's diversity combines richness (i.e., the number of feature types present) and evenness (i.e., the distribution of area among features). Cohesion index (CI) was used to quantify connectivity among land cover features within a patch (Schumaker 1996). Proximity/isolation was measured by Euclidean nearest neighbor distance (McGarigal et al. 2002). All of the metrics, except for Euclidean nearest neighbor distance, were calculated at both the feature level (e.g., PD_Build, patch density for building) and whole-patch level (e.g., PD_LS, patch density of all five land cover features within a patch) (Table 2). Statistical summaries, including mean and standard deviation, were calculated for metrics of patch size, patch edge, fractal dimension index, shape index, perimeter-area ratio, and Euclidean nearest neighbor distance (Table 2).

Metrics of whole-patch characteristics and withinpatch composition and configuration were calculated for each feature separately in ArcGIS ${ }^{\mathrm{TM}} 9.3$ (McGarigal et al. 2002). These metrics were calculated based on the HERCULES patch layer and the reference land cover layer generated from object-based classification (Table 2). These metrics were used as predictor variables in later statistical analyses to examine whether whole-patch and within-patch characteristics affect the accuracy of cover estimates based on visual interpretation.

\section{Statistical analyses}

Using logistic regressions, we first examined how variables of whole-patch characteristics could affect cover estimates by visual interpretation. We repeated the analyses using the variables of within-patch composition and configuration. Whether a combination of whole-patch and within-patch variables yielded better predictions than either alone was then investigated. The response variable for each of the five land cover features was binary, with value of one or zero representing the proportion cover estimates from visual interpretation were correct or incorrect. Five models were constructed and compared for each response variable. Those five models have a given dependent variable as a function of: (1) whole-patch 


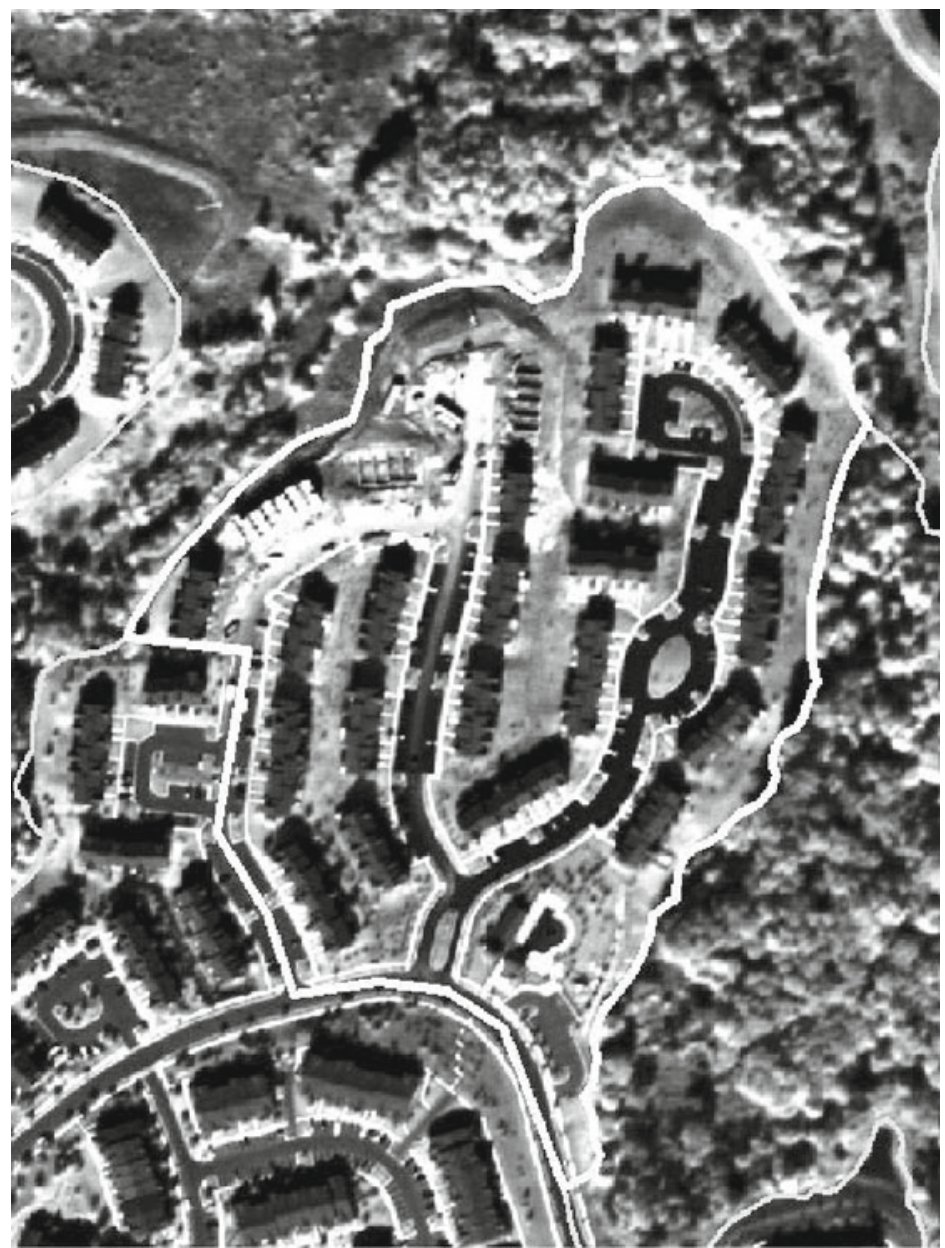

Variables Value

Metrics of whole-patch characteristics

PA (ha) $\quad 7.28$

PERIM (km) $\quad 1.24$

FRAC $\quad 1.03$

PARA $\quad 0.02$

SHAPE $\quad 1.15$

Metrics of within-patch composition

Per_Build $\quad 0.16$

Per_CV $\quad 0.03$

Per_FV $\quad 0.24$

Per_Pave $\quad 0.47$

Per_BS 0.10

SIDI $\quad 0.69$

Metrics of within-patch configuration

AMN_Build $\left(\mathrm{m}^{2}\right) \quad 493.9$

EMN_Build (m) $\quad 159.5$

LPI_Build $\quad 1.43$

PD_Build $\quad 329.6$

ED_Build $\quad 525.8$

PARAMN_Build $\quad 0.36$

SHAPEMN_Build $\quad 1.81$

FRACMN_Build $\quad 1.19$

ENN_MN_Build (m) 35.8

CI_Build 97.6

AMN_LS $\left(\mathrm{m}^{2}\right) \quad 353.5$

EMN_LS (m) 110.6

LPI_LS 43.4

PD_LS $\quad 2929.2$

ED_LS $\quad 3127.8$

PARAMN_LS $\quad 1.36$

SHAPEMN_LS $\quad 1.67$

FRACMN_LS $\quad 1.24$

CI_LS $\quad 99.42$

Fig. 3 An example of a HERCULES patch (highlighted), and the values of the metrics that measure the whole-patch and within-patch characteristics. The building feature is used as an example in the table (Note: not all of the configuration metrics for building are listed)

characteristics; (2) within-patch composition; (3) within-patch configuration; (4) within-patch composition + within-patch configuration; and (5) wholepatch characteristics + within-patch composition + within-patch configuration (Table 3 ). Because there were a large number of predictor variables, and some were highly correlated to each other, we used a forward stepwise variable selection procedure in the logistic regressions to determine which variables to add or drop from the models (Hosmer and Lemeshow 2000). The entry probability was set as 0.05 . Consequently, only significant predictor variables were kept in the final models.

In a logistic regression, a response variable that is typically binary $(0,1)$ is predicted as a function of a series of continuous or categorical predictor variables. Rather than model the binary response variable directly, logistic regression converts the response variable into a logit variable, or the natural log odds of the response occurring. The logistic regression model is given as (Agresti 1996):

$$
\begin{aligned}
\operatorname{logit}(p) & =\ln (p /(1-p)) \\
& =\alpha+\beta_{1} x_{1}+\beta_{2} x_{2}+\cdots+\beta_{k} x_{k}
\end{aligned}
$$

where $\ln$ is the natural logarithm, $p$ is the probability of the proportion cover of a feature within a patch (e.g., buildings) being correctly classified, $p /(1-p)$ is the odds of a specific response occurring, $\alpha$ is the intercept, $x_{1}$ through $x_{\mathrm{k}}$ are predictor variables, and $\beta_{n}$ is the coefficient of variable $x_{\mathrm{n}}$.

A positive regression coefficient indicates that the odds of a correct classification increases as the predictor variable increases, while a negative regression coefficient means a decrease in the odds of a 
Table 2 Metrics of whole-patch characteristics and within-patch composition and configuration of land cover features

\begin{tabular}{|c|c|c|c|}
\hline Variable & Description & Mean & SD \\
\hline \multicolumn{4}{|c|}{ Metrics of whole-patch characteristics } \\
\hline $\mathrm{PA}$ & Patch area $\left(\mathrm{m}^{2}\right)$ & 7.62E4 & $2.38 \mathrm{E} 5$ \\
\hline PERIM & Patch perimeter $(\mathrm{m})$ & 1303.88 & 2189.32 \\
\hline FRAC & Fractal dimension index, measure of shape complexity & 1.046 & 0.044 \\
\hline PARA & Perimeter-area ratio, measure of shape complexity & 0.043 & 0.314 \\
\hline SHAPE & Shape index, measure of shape complexity & 1.332 & 0.532 \\
\hline \multicolumn{4}{|c|}{ Metrics of within-patch composition of land cover features } \\
\hline Per_Build & Percent buildings & 0.117 & 0.120 \\
\hline Per_CV & Percent CV & 0.261 & 0.248 \\
\hline Per_FV & Percent FV & 0.306 & 0.225 \\
\hline Per_Pave & Percent pavement & 0.278 & 0.208 \\
\hline Per_BS & Percent bare soil & 0.032 & 0.122 \\
\hline SIDI & Simpson's diversity index & 0.558 & 0.175 \\
\hline \multicolumn{4}{|c|}{ Metrics of within-patch configuration of land cover features } \\
\hline AMN_Build & Mean patch sizes of all building patches & 577.15 & 1425.69 \\
\hline AMN_LS & Mean of patch sizes of all patches & 690.30 & 675.01 \\
\hline ASD_Build & Standard deviation of patch sizes of all building patches & 452.79 & 1407.93 \\
\hline ASD_LS & Standard deviation of patch sizes of all patches & 3073.87 & 4825.72 \\
\hline EMN_Build & Mean of patch edges of all building patches & 110.47 & 131.80 \\
\hline EMN_LS & Mean of patch edges of all patches & 158.95 & 61.97 \\
\hline ESD_Build & Standard deviation of edges of all building patches & 61.35 & 104.54 \\
\hline ESD_LS & Standard deviation of edges of all patches & 434.52 & 296.33 \\
\hline LPI_Build & Largest patch index for building, calculated at feature level & 5.02 & 8.25 \\
\hline LPI_LS & Largest patch index calculated at landscape level & 48.08 & 21.63 \\
\hline PD_Build & Patch density of buildings, the number of building patches per $\mathrm{km}^{2}$ & 338.09 & 518.72 \\
\hline PD_LS & Patch density, the number patches per $\mathrm{km}^{2}$ & 6451.92 & $1.93 \mathrm{E} 5$ \\
\hline ED_Build & Edge density of buildings, the total length of all building patches per hectare & 321.21 & 313.22 \\
\hline ED_LS & Edge density, the total length of all patches per hectare & 3131.58 & 3282.66 \\
\hline PARAMN_Build & Mean of PARA of all building patches & 1.50 & 10.63 \\
\hline PARAMN_LS & Mean of PARA of all patches & 2.38 & 3.22 \\
\hline PARASD_Build & Standard deviation of PARA of all building patches & 1.67 & 11.74 \\
\hline PARASD_LS & Standard deviation of PARA of all patches & 5.54 & 14.79 \\
\hline SHAPEMN_Build & Mean of SHAPE of all building patches & 1.54 & 0.64 \\
\hline SHAPE MN_LS & Mean of SHAPE of all patches & 1.99 & 0.29 \\
\hline SHAPE SD_Build & Standard deviation of SHAPE of all building patches & 0.27 & 0.50 \\
\hline SHAPESD_LS & Standard deviation of SHAPE of all patches & 1.07 & 0.33 \\
\hline FRACMN_Build & Mean of FRAC of all building patches & 1.15 & 0.15 \\
\hline FRACMN_LS & Mean of FRAC of all patches & 1.29 & 0.06 \\
\hline FRACSD_Build & Standard deviation of FRAC of all building patches & 0.073 & 0.11 \\
\hline FRACSD_LS & Standard deviation of FRAC of all patches & 0.21 & 0.05 \\
\hline ENN_MN_Build & Mean of Euclidean nearest neighboring distance, an isolation metric & 29.72 & 30.34 \\
\hline ENN_SD_Build & Standard deviation of Euclidean nearest neighbor distance & 9.35 & 13.80 \\
\hline CI_Build & Patch cohesion index at the feature level for building patches & 77.76 & 37.88 \\
\hline
\end{tabular}


Table 2 continued

\begin{tabular}{llrl}
\hline Variable & Description & Mean & SD \\
\hline CI_LS & Patch cohesion index at the landscape level & 99.22 & 0.44 \\
\hline
\end{tabular}

The building feature is used as an example in this table. These metrics were used as predictor variables. For each feature, the predictors of whole-patch characteristics, within-patch composition, and within-patch configuration at the whole patch level were the same as listed in the table, but the predictors of within-patch configuration at the feature level are different. For example, for CV, the patch density of CV (PD_CV), rather than that of Building (PD_Build), was used

Table 3 Logistic regression models compared for each of the five land cover features

\begin{tabular}{lll}
\hline Model number & Model & Description \\
\hline 1 & $\alpha+\beta_{1} X_{1}$ & Whole-patch characteristics \\
2 & $\alpha+\beta_{2} X_{2}$ & Within-patch composition \\
3 & $\alpha+\beta_{3} X_{3}$ & Within-patch configuration \\
4 & $\alpha+\beta_{2} X_{2}+\beta_{3} X_{3}$ & Within-patch composition + within-patch configuration \\
5 & $\alpha+\beta_{1} X_{1}+\beta_{2} X_{2}+\beta_{3} X_{3}$ & Whole-patch characteristics + within-patch composition + within-patch configuration
\end{tabular}

For each land cover feature, the response variable was binary, with value of one or zero indicating whether or not the proportion cover estimates from visual interpretation were in the same category as those generated from the object-based approach. $X_{1}$ is the vector of whole-patch characteristics variables, $X_{2}$ is the vector of within-patch composition variables, $X_{3}$ is the vector of within-patch configuration variables, $\alpha$ is the intercept, and each $\beta$ represents a coefficient vector

correct classification when the predictor variable increases. For continuous predictor variables, the magnitude of $\beta_{n}$ is interpreted as the additive effect on the log odds ratio for a unit change in the predictor variable $x_{\mathrm{n}}$, holding all else constant (Agresti 1996).

Multi-model inferential procedures were used for model comparisons to determine which of the wholepatch or within-patch variables, or some combination, best predicts the variance in each response variable (i.e., correct/incorrect classification of each feature) (Burnham and Anderson 2002). This procedure, which is based on minimization of Akaike's Information Criterion (AIC) (Akaike 1973), selects the model that best explains the data with the fewest parameters. We also calculated the Akaike weight for each model, or the probability of a given model being the best one among a number of candidate models. Akaike weights are especially useful when the difference of AIC values between two models is small (Burnham and Anderson 2002; Wagenmakers and Farrell 2004). Separate comparisons were run for each response variable.

\section{Results}

Five models were compared for each response variable, and we present the model parameters, pseudo $R^{2}$ value, AIC value, and Akaike weight for each model (Tables $4,5,6,7,8)$. The pseudo $R^{2}$ value is a measure of the strength of association for regression models containing a categorical dependent variable (Nagelkerke 1991). It is similar to the coefficient of determination, $R^{2}$, for linear regression models. For all models within each model group (i.e., the same binary response variable), we ranked each model on the basis of its AIC value. Below, we first present the results from model comparisons for each of the five features. We then discuss the effects of whole-patch characteristics and within-patch composition and configuration of features on the accuracy of cover estimates.

Model comparison: the best model for each land cover feature

\section{Building, coarse-textured vegetation, and bare soil}

The best models for these three features are the most complex models which combine variables of wholepatch characteristics, and within-patch composition and configuration (Tables $4,5,8$ ). For example, the best model to predict the accuracy of estimating the cover of buildings, Build5, combined a whole-patch characteristic (patch perimeter), within-patch composition 
Table 4 Summary results of logistic regression models for the building land cover, including AIC scores, Akaike weights, model ranking and pseudo $R^{2}$ values

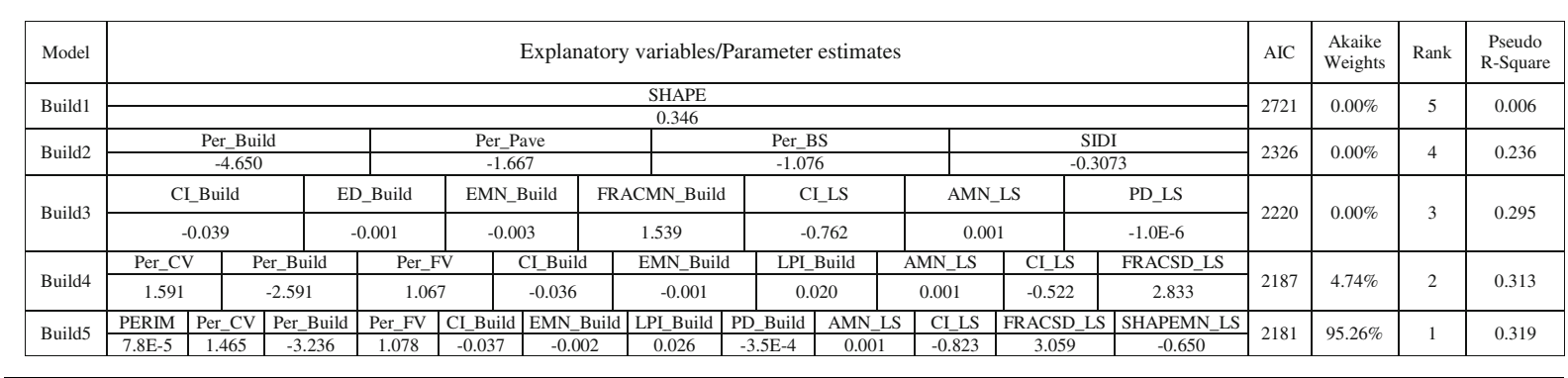

Only significant predictor variables were kept in the models

Table 5 Summary results of logistic regression models for the coarse textured vegetation land cover, excluding insignificant terms

\begin{tabular}{|c|c|c|c|c|c|c|c|c|c|c|c|c|c|c|c|c|c|}
\hline Model & \multicolumn{13}{|c|}{ Explanatory variables/Parameter estimates } & AIC & $\begin{array}{c}\text { Akaike } \\
\text { Weights }\end{array}$ & Rank & $\begin{array}{c}\text { Pseudo } \\
\text { R-Square }\end{array}$ \\
\hline \multirow{3}{*}{$\begin{array}{l}\mathrm{CV} 1 \\
\mathrm{CV} 2 \\
\end{array}$} & \multicolumn{7}{|c|}{$\begin{array}{l}\text { PARA } \\
-10.22\end{array}$} & \multicolumn{6}{|c|}{$\begin{array}{l}\text { PERIM } \\
1.01 \mathrm{E}-4 \\
\end{array}$} & 2961 & $0.00 \%$ & 5 & 0.031 \\
\hline & \multirow{2}{*}{\multicolumn{5}{|c|}{$\begin{array}{l}\text { SIDI } \\
-2.63\end{array}$}} & \multirow{2}{*}{\multicolumn{4}{|c|}{$\frac{P \text { Per_FV }}{-1.12}$}} & \multirow{2}{*}{\multicolumn{4}{|c|}{$\begin{array}{l}\text { Per_Build } \\
2.24\end{array}$}} & 2897 & $000 \%$ & 4 & 0.069 \\
\hline & & & & & & & & & & & & & & \multirow[b]{3}{*}{2762} & & & \\
\hline \multirow[b]{2}{*}{ CV3 } & AMN_CV & ENNM & & & D_CV & ED_CV & ASD_CV & EMN_CV & ASD_LS & LPI_LS & EMN_L & & HAPEMN_LS & & \multirow[b]{2}{*}{$0.05 \%$} & \multirow[b]{2}{*}{3} & \multirow[b]{2}{*}{0.152} \\
\hline & $4.04 \mathrm{E}-4$ & -0.0 & & & 0.001 & -0.001 & $-5.5 \mathrm{E}-5$ & -0.003 & $9.9 \mathrm{E}-5$ & -0.007 & -0.003 & & -0.521 & & & & \\
\hline \multirow{2}{*}{ CV4 } & Per_CV & Per_E & & & MN_CV & ED_CV & ESD_CV & PD_CV & AMN_CV & $\begin{array}{l}\text { LPI_CV } \\
\end{array}$ & ASD_L & & HAPEMN_LS & \multirow{2}{*}{2756} & \multirow{2}{*}{$1.10 \%$} & \multirow{2}{*}{2} & \multirow{2}{*}{0.155} \\
\hline & 3.08 & 1.8 & & & 0.024 & -0.001 & $-4.62 \mathrm{E}-4$ & -0.001 & $1.41 \mathrm{E}-4$ & -0.018 & $7.3 \mathrm{E}-5$ & & -0.573 & & & & \\
\hline CV5 & $\begin{array}{c}\text { PARA } \\
-14.055\end{array}$ & $\begin{array}{l}\text { PA } \\
1 \mathrm{E}-6\end{array}$ & $\frac{5}{-2}$ & $\begin{array}{l}\text { DI } \\
065\end{array}$ & $\frac{\text { Per_Build }}{2.145}$ & $\frac{P e r_{C} C V}{1.818}$ & $\begin{array}{c}\text { Per_Pave } \\
0.683\end{array}$ & $\begin{array}{c}\text { ENNMN_CV } \\
-0.024\end{array}$ & $\begin{array}{c}\text { ED_CV } \\
-0.001\end{array}$ & $\begin{array}{l}\text { ESD_CV } \\
-4.73 \mathrm{E}-4\end{array}$ & $\frac{\overline{A M N} \_C V}{1.31 E-4}$ & $\begin{array}{l}\text { PD_CV } \\
-4.25 \mathrm{E}-4\end{array}$ & \begin{tabular}{l|l}
$\mathrm{V}$ & ED_LS \\
-4 & $2.45 \mathrm{E}-4$
\end{tabular} & 2747 & $98.85 \%$ & 1 & 0.162 \\
\hline
\end{tabular}

Table 6 Summary results of logistic regression models for the fine textured vegetation land cover, excluding insignificant terms

\begin{tabular}{|c|c|c|c|c|c|c|c|c|c|c|c|}
\hline Model & \multicolumn{7}{|c|}{ Explanatory variables/Parameter estimates } & AIC & $\begin{array}{c}\text { Akaike } \\
\text { Weights }\end{array}$ & Rank & $\begin{array}{l}\text { Pseudo } \\
\text { R-Square }\end{array}$ \\
\hline FV1 & \multicolumn{7}{|c|}{$\begin{array}{c}\text { PARA } \\
-6.81\end{array}$} & 3086 & $000 \%$ & 5 & 0007 \\
\hline \multirow{2}{*}{ FV2 } & \multirow{2}{*}{\multicolumn{2}{|c|}{$\begin{array}{c}\text { Per_Build } \\
3.567\end{array}$}} & \multirow{2}{*}{\multicolumn{2}{|c|}{$\frac{P e r \_C V}{-0.928}$}} & \multirow{2}{*}{\multicolumn{3}{|c|}{$\frac{\text { Per_FV }}{0.893}$}} & \multirow[b]{2}{*}{2984} & \multirow[b]{2}{*}{$0.00 \%$} & \multirow[b]{2}{*}{4} & \multirow[b]{2}{*}{0.069} \\
\hline & & & & & & & & & & & \\
\hline \multirow{2}{*}{ FV3 } & \multicolumn{2}{|c|}{ AMN_FV } & ED_FV & EMN_FV & \multicolumn{2}{|c|}{ LPI_LS } & EMN_LS & \multirow{2}{*}{2972} & \multirow{2}{*}{$0.00 \%$} & \multirow{2}{*}{3} & \multirow{2}{*}{0.077} \\
\hline & \multicolumn{2}{|l|}{$5.22 \mathrm{E}-4$} & -0.001 & -0.002 & \multicolumn{2}{|c|}{-0.019} & -0.003 & & & & \\
\hline \multirow{2}{*}{ FV4 } & Per_CV & Per_Build & SIDI & AMN_FV & EMN_FV & LPI_LS & ASD_LS & \multirow{2}{*}{2921} & \multirow{2}{*}{$62.25 \%$} & \multirow{2}{*}{1} & \multirow{2}{*}{0.107} \\
\hline & -1.264 & 2.416 & -2.176 & $4.28 \mathrm{E}-4$ & -0.003 & -0.022 & $3.0 \mathrm{E}-5$ & & & & \\
\hline FV5 & PARA & PERIM & $\frac{\text { Per_CV }}{1.45}$ & $\begin{array}{l}\text { Per_Build } \\
1974\end{array}$ & $\frac{\text { AMN_FV }}{4 \text { FE }^{2}}$ & EMN_FV & ESD_LS & 2922 & $37.75 \%$ & 2 & 0.107 \\
\hline
\end{tabular}

(percent of $\mathrm{CV}$, percent of building, and percent of $\mathrm{FV}$ ) and within-patch configuration (e.g., connectivity among buildings, building density and shape complex) (Table 4). Approximately $32 \%$ of the variation in the response variable of building cover accuracy was explained by this model.

\section{Fine-textured vegetation}

The best model (FV4) for this feature combines variables of within-patch composition and configuration (Table 6). The second best model (FV5) combines variables of whole-patch characteristics and 
Table 7 Summary results of logistic regression models for the pavement land cover, excluding insignificant terms

\begin{tabular}{|c|c|c|c|c|c|c|c|c|c|c|c|c|c|c|c|c|}
\hline Model & \multicolumn{12}{|c|}{ Explanatory variables/Parameter estimates } & AIC & $\begin{array}{c}\text { Akaike } \\
\text { Weights }\end{array}$ & Rank & $\begin{array}{l}\text { Pseudo } \\
\text { R-Square }\end{array}$ \\
\hline \multirow{2}{*}{ Pave1 } & \multirow{2}{*}{\multicolumn{6}{|c|}{$\begin{array}{l}\text { PARA } \\
-9.282 \\
\end{array}$}} & \multirow{2}{*}{\multicolumn{6}{|c|}{$\begin{array}{c}\text { SHAPE } \\
0.287\end{array}$}} & 3085 & $0.00 \%$ & 5 & 0.020 \\
\hline & & & & & & & & & & & & & 3085 & $0.00 \%$ & 3 & 0.020 \\
\hline \multirow{2}{*}{ Pave2 } & \multicolumn{6}{|c|}{ SIDI } & \multicolumn{6}{|c|}{ Per_BS } & 2974 & $0.00 \%$ & 4 & 0.083 \\
\hline & \multicolumn{6}{|c|}{-2.953} & \multicolumn{6}{|c|}{-1.504} & $29 / 4$ & $0.00 \%$ & 4 & 0.083 \\
\hline \multirow{2}{*}{ Pave3 } & ED_Pave & LPI & & \multirow{2}{*}{\multicolumn{2}{|c|}{$\begin{array}{c}\text { SHAPESD_Pave } \\
-0.221\end{array}$}} & CI_Pave & ENNSD & ave & FRACMI & Pave & CI_LS & ESD_LS & \multirow{2}{*}{2810} & \multirow[b]{2}{*}{$0.00 \%$} & \multirow[b]{2}{*}{3} & \multirow{2}{*}{0.177} \\
\hline & -0.002 & 0.0 & & & & -0.024 & 0.01 & & 1.02 & & -0.532 & $-8.65 \mathrm{E}-4$ & & & & \\
\hline \multirow{2}{*}{ Pave4 } & Per_Pave & Per_BS & & Build & SIDI & ED_Pave & PD_Pave & FRA & MN_Pave & CI_Pave & FRACSD_LS & FRACMN_LS & \multirow{2}{*}{2773} & \multirow{2}{*}{$50.00 \%$} & \multirow[b]{2}{*}{1} & \multirow{2}{*}{0.199} \\
\hline & 3.531 & -2.164 & & 907 & -1.195 & -0.003 & $-5.22 \mathrm{E}-4$ & & 894 & -0.020 & -5.498 & 3.461 & & & & \\
\hline \multirow{2}{*}{ Pave5 } & Per_Pave & Per_BS & & Build & SIDI & ED_Pave & PD_Pave & FRA & MN_Pave & CI_Pave & FRACSD_LS & FRACMN_LS & \multirow{2}{*}{2773} & \multirow{2}{*}{$50.00 \%$} & \multirow{2}{*}{1} & \multirow{2}{*}{0.199} \\
\hline & 3.531 & -2.164 & & 907 & -1.195 & -0.003 & $-5.22 \mathrm{E}-4$ & & 894 & -0.020 & -5.498 & 3.461 & & & & \\
\hline
\end{tabular}

Table 8 Summary results of logistic regression models for the bare soil land cover, excluding insignificant terms

\begin{tabular}{|c|c|c|c|c|c|c|c|c|c|c|c|}
\hline Model & \multicolumn{7}{|c|}{ Explanatory variables/Parameter estimates } & AIC & $\begin{array}{c}\text { Akaike } \\
\text { Weights }\end{array}$ & Rank & $\begin{array}{c}\text { Pseudo } \\
\text { R-Square }\end{array}$ \\
\hline $\mathrm{BS} 1$ & \multicolumn{7}{|c|}{ None of the variables is significant } & \multirow{2}{*}{2206} & \multirow{2}{*}{$0.00 \%$} & \multirow{2}{*}{5} & \multirow[b]{2}{*}{0} \\
\hline BSI & \multicolumn{7}{|c|}{ N/A } & & & & \\
\hline \multirow[b]{2}{*}{ BS2 } & Per_Build & \multirow{2}{*}{\multicolumn{2}{|c|}{$\begin{array}{c}\text { Per_CV } \\
1.427 \\
\end{array}$}} & \multirow{2}{*}{$\begin{array}{c}\text { Per_Pave } \\
-1.257\end{array}$} & & \multirow{2}{*}{$\begin{array}{c}\text { Per_BS } \\
-2.448\end{array}$} & SIDI & \multirow[b]{2}{*}{2084} & \multirow[b]{2}{*}{$0.00 \%$} & \multirow[b]{2}{*}{4} & \multirow[b]{2}{*}{0.091} \\
\hline & 2.955 & & & & & & -1.34 & & & & \\
\hline \multirow{2}{*}{ BS3 } & ED_BS & \multicolumn{2}{|c|}{ SHAPEMN_BS } & \multicolumn{2}{|c|}{ ENN_MN_BS } & ENN_SD_BS & AMN_BS & \multirow{2}{*}{2051} & \multirow{2}{*}{$0.00 \%$} & \multirow{2}{*}{3} & \multirow{2}{*}{0.113} \\
\hline & -0.002 & \multicolumn{2}{|c|}{-0.244} & -0.003 & & -0.012 & $2.2 \mathrm{E}-5$ & & & & \\
\hline \multirow{2}{*}{ BS4 } & Per_Pave & Per_FV & & & SHAPEMN_BS & ENN_MN_BS & ENN_SD_BS & \multirow{2}{*}{1988} & \multirow{2}{*}{$2.93 \%$} & \multirow[b]{2}{*}{2} & \multirow{2}{*}{0.155} \\
\hline & -2.687 & $-1 . \overline{3} 61$ & & & -0.267 & -0.003 & -0.013 & & & & \\
\hline \multirow{2}{*}{ BS5 } & PERIM & Per_pave & Per_FV & ED_BS & SHAPEMN_BS & ENN_MN_BS & ENN_SD_BS & \multirow{2}{*}{1981} & \multirow{2}{*}{$97.7 \%$} & \multirow[b]{2}{*}{1} & \multirow{2}{*}{0.161} \\
\hline & $1.18 \mathrm{E}-4$ & -2.649 & -1.270 & -0.002 & -0.303 & -0.003 & -0.017 & & & & \\
\hline
\end{tabular}

within-patch composition and configuration. Though AIC and Akaike weights indicate that FV4 is slightly better than FV5, support for FV4 being the best relative to FV5 is weak.

\section{Pavement}

The model that best explains pavement (Pave4) combines variables of within-patch composition and configuration (Table 7). After adjusting for the effects of within-patch composition and configuration, no whole-patch characteristic significantly contributes to the explanation of variance in pavement cover estimates (Pave5). Therefore, the model Pave4 was identical to the model Pave5.

The best model for each of the five land cover features provides a better prediction than that by the baseline or intercept-only model (Table 9). The intercept-only model classifies all cases simply by using the most numerous category (i.e., the category 0). For example, the best model for pavement (Pave5) classifies $68.7 \%$ correctly compared to $52.5 \%$ classified correctly by the intercept-only model. For each land cover feature, combining within-patch composition variables with those of within-patch configuration (model group 4) provides better prediction than those models using variables of within-patch composition or configuration alone (model group 2 or 3 ).

The effects of whole-patch characteristics on the accuracy of cover estimates

Whole-patch characteristics are significant predictors for all five land cover features. Models using patch characteristics alone (i.e., model group 1) provide statistically significant improvement over the intercept-only models, except for bare soil (model BS1, Table 8). However, little variance in estimation accuracy was explained by this model group, suggesting that whole-patch characteristics alone do not provide adequate explanation.

Among the five indicators of whole-patch characteristics, perimeter-area ratio $(P A R A)$ and perimeter (PERIM) are the most significant. PARA was a significant predictor for $\mathrm{CV}$ and $\mathrm{FV}$ when adjusting for the effects of variables of within-patch composition 
Table 9 Classification table for the best models and baseline or intercept-only models summarized by land cover features

\begin{tabular}{|c|c|c|c|c|c|c|}
\hline \multirow[t]{2}{*}{ Landscape feature } & \multirow[t]{2}{*}{ Model } & \multirow[t]{2}{*}{ Observed } & \multicolumn{2}{|c|}{ Predicted } & \multirow{2}{*}{$\begin{array}{l}\text { Percent } \\
\text { correct }(\%)\end{array}$} & \multirow{2}{*}{$\begin{array}{l}\text { Overall } \\
\text { percent correct }(\%)\end{array}$} \\
\hline & & & 1 & 0 & & \\
\hline \multirow[t]{4}{*}{ Building } & \multirow[t]{2}{*}{ Best } & 1 & 1,413 & 176 & 88.9 & \multirow[t]{2}{*}{73.6} \\
\hline & & 0 & 417 & 244 & 36.9 & \\
\hline & \multirow[t]{2}{*}{ Intercept-only } & 1 & 1,589 & 0 & 100 & \multirow[t]{2}{*}{70.6} \\
\hline & & 0 & 662 & 0 & 0 & \\
\hline \multirow[t]{4}{*}{$\mathrm{CV}$} & \multirow[t]{2}{*}{ Best } & 1 & 1,156 & 219 & 64.0 & \multirow[t]{2}{*}{64.8} \\
\hline & & 0 & 572 & 303 & 72.9 & \\
\hline & \multirow[t]{2}{*}{ Intercept-only } & 1 & 1,375 & 0 & 100 & \multirow[t]{2}{*}{61.1} \\
\hline & & 0 & 875 & 0 & 0 & \\
\hline \multirow[t]{4}{*}{$\mathrm{FV}$} & \multirow[t]{2}{*}{ Best } & 1 & 935 & 310 & 75.1 & \multirow[t]{2}{*}{61.6} \\
\hline & & 0 & 555 & 450 & 44.8 & \\
\hline & \multirow[t]{2}{*}{ Intercept-only } & 1 & 1,245 & 0 & 100 & \multirow[t]{2}{*}{55.3} \\
\hline & & 0 & 1,005 & 0 & 0 & \\
\hline \multirow[t]{4}{*}{ Pavement } & \multirow[t]{2}{*}{ Best } & 1 & 683 & 385 & 64.0 & \multirow[t]{2}{*}{68.7} \\
\hline & & 0 & 320 & 862 & 72.9 & \\
\hline & \multirow[t]{2}{*}{ Intercept-only } & 1 & 0 & 1,068 & 0 & \multirow[t]{2}{*}{52.5} \\
\hline & & 0 & 0 & 1,182 & 100 & \\
\hline \multirow[t]{4}{*}{ Bare soil } & \multirow[t]{2}{*}{ Best } & 1 & 1,769 & 48 & 97.4 & \multirow[t]{2}{*}{81.4} \\
\hline & & 0 & 371 & 62 & 14.3 & \\
\hline & \multirow[t]{2}{*}{ Intercept-only } & 1 & 1,817 & 0 & 100 & \multirow[t]{2}{*}{80.8} \\
\hline & & 0 & 433 & 0 & 0 & \\
\hline
\end{tabular}

The columns are the two predicted values of the dependent, while the rows are the two observed (actual) values of the dependent. One means that the cover of the feature was correctly estimated, while zero represents misclassification. The values on the diagonal of the two columns under 'predicted' (e.g., 1413 and 244, marked in grey) are the number of cases correctly classified by the models. The column "percent correct" is the percentage of cases correctly classified for a specific category (i.e., 1 or 0 ), while the "overall percent correct" is the total percentage of cases correctly classified

and configuration (CV5 and FV5). The negative coefficients of PARA indicated that the odds of a correct estimation decrease with the increase of patch PARA. Patch perimeter (PERIM) was a significant predictor for building, FV, and bare soil, when the effects of variables of within-patch composition and configuration were adjusted (Build5, FV5, and BS5). The positive parameters of PERIM in the three best models indicated that the odds of a correct estimation for those three features increase with the increase of PERIM.

The effects of within-patch composition on the accuracy of cover estimates

Variables of within-patch composition are better predictors of estimation accuracy than those of whole-patch characteristics for all of the five land cover features. Among the six variables of withinpatch composition, the cover of buildings (Per_Build) and the Simpson's diversity index (SIDI) are the most significant metrics predicting the accuracy. SIDI was significant in all five models, but SIDI was only significant for pavement when the effects of wholepatch characteristics and within-patch configuration were adjusted. The general effects of SIDI were consistent for all five land cover features meaning that the odds of correctly estimating cover decrease with the increase of the diversity of land cover features. However, the estimated coefficients, or the magnitudes of the impacts of SIDI, varied broadly among features.

Proportion cover of buildings within the patch (Per_Build) significantly affected the prediction of accuracy for all of the land cover features except for bare soil. It remained significant in all the best models 
for $\mathrm{FV}, \mathrm{CV}$, pavement and building features, when effects of whole-patch characteristics and withinpatch configuration were adjusted. The effects of Per_Build on the accuracy, however, varied by feature. While the negative coefficient of Per_Build indicated that the increase of building cover within a patch decreased the odds of a correct estimation for building cover, the positive coefficients of Per_Build for $\mathrm{CV}, \mathrm{FV}$ and pavement indicated that the odds of a correct estimation for those land cover features increased with the increase of building cover within a patch.

The effects of within-patch configuration on the accuracy of cover estimates

Models using variables of configuration (model group 3) are superior to those using composition variables alone (model group 2), as well as models only using variables of whole-patch characteristics (model group 1). Configuration metrics, both at the feature level and the whole-patch level, affected the accuracy of cover estimates (e.g., cohesion index at both levels, CI_Build and CI_LS in model Build3, Table 4). The significance of configuration variables varied broadly among features, with only a very few variables being significant for all or most of the five land cover features. Significant variables included edge density, mean patch size, and mean edge length, all at the feature level. While the significance of configuration variables varied by features, the effects of the same configuration variable were generally consistent across all land cover features. For example, in the model group using only within-patch configuration variables as predictors (model group 3), variables of edge density (e.g., ED_Build) were significant for all of the five land cover features, and had consistent negative effects on the accuracy. This suggests that for each of the five features, the odds of the cover of that feature being correctly estimated decreased with an increase of edge density.

\section{Discussion}

The results indicate that, though the accuracy of visually estimating the cover of features within patches is significantly affected by both whole-patch characteristics and within-patch composition and configuration of features, within-patch configuration is the most significant factor. In addition, within-patch composition is a better predictor than whole-patch characteristics. Most frequently, however, a combination of whole-patch and within-patch characteristics provides the best prediction of accuracy of cover estimates. The relative importance of whole-patch characteristics, and within-patch composition and configuration on the accuracy of cover estimates will be discussed in order of increasing importance.

Whole-patch characteristics

Characteristics, such as perimeter and perimeter-area ratio, are useful predictors of accuracy of cover estimates through visual interpretation, even after the effects of within-patch composition and configuration were adjusted. The statistical importance of these whole-patch variables indicates that some of the variability in the accuracy of cover estimates that is not explained by within-patch composition and configuration can be attributed to whole-patch characteristics. A very small proportion of the variance in the accuracy of cover estimates, however, can be explained by whole-patch characteristics alone. Our results suggest errors in cover estimates are more likely to occur for smaller patches with more complex shapes, which have larger perimeter to area ratios. This is similar to previous research that digitized individual landscape features and found that misclassification was more likely to occur for smaller features with higher perimeter to area ratios (Ellis and Wang 2006).

\section{Within-patch composition}

Composition of land cover features within a patch is a better predictor of accuracy through visual interpretation than whole-patch characteristics. Both the proportion abundance of each land cover feature and the diversity of features within a patch significantly affect the accuracy of cover estimates. The most important composition variable in predicting this accuracy is the percent cover of buildings. As the percent cover of buildings increases, the accuracy of estimating building cover decreases, but the accuracy of estimating coarse-textured vegetation $(\mathrm{CV})$, fine-textured vegetation (FV), and pavement increases. Our results suggest that errors in estimating cover are more likely 
to occur when within-patch composition is more diverse. This is consistent with findings from previous studies that visual estimates of woody crown cover from aerial photography may be influenced by land type (Fensham et al. 2002; Fensham and Fairfax 2007), and that the accuracy of digital land cover classification tends to decrease with the increase of diversity in land cover features (Smith et al. 2003).

Effects of the relative cover of different land cover features on the accuracy of estimating the cover of those features vary by feature types. For example, errors in estimating cover of $\mathrm{CV}$ are more likely to occur as the relative amount of $\mathrm{CV}$ within a patch decreases. Errors in estimating building cover, however, are more likely to occur with higher building cover. This difference may suggest that interpreters perceive and react to patterns of built (i.e., building) and non-built (e.g. CV) components in different ways.

Within-patch configuration

Within-patch configuration is the most significant predictor of accuracy of cover estimates through visual interpretation. A large number of variables contribute to this significance. While the significance and magnitudes of effects of the variables vary broadly among different types of land cover features, the effects were generally consistent. Our results suggest that errors of cover estimates are more likely to occur when land cover features within a patch are (1) more fragmented (e.g., higher patch density, higher edge density, smaller averaged patch size, and smaller largest patch index); (2) more complex in shape (e.g., patches are more irregular, with larger values of shape index), and (3) physically less connected (e.g., larger averaged nearest neighbor distance). These results may be due to how interpreters perceive and react to landscape patterns by applying many of the Gestaltlaws simultaneously during the process of visual interpretation (Antrop and Van Eetvelde 2000). For example, interpreters generally reduce the complexity of pattern they observe by transforming irregular shapes into geometric shapes and grouping them according to similarity and proximity (Antrop and Van Eetvelde 2000). Consequently, this simplification and interpretation may lead to the decrease in accurately estimating cover, with the increase in complexity of features configuration within a patch. Because the urban landscape is strikingly heterogeneous, with complex fine-scale spatial patterning of individual features such as buildings, driveways and lawns, visual interpretation may not be an effective way to accurately estimate cover of land cover features within a patch (Zhou et al. 2010). This limitation suggests that other approaches, for example object-based image analysis, that can accurately estimate cover of features within a patch are desirable (Benz et al. 2004; Zhou and Troy 2008; Blaschke 2010).

Whole-patch and within-patch characteristics are important predictors of the accuracy of cover estimates based on visual interpretation. The relatively low pseudo $R^{2}$ values of the regression models, however, suggest that models using variables of whole-patch and within-patch characteristics as predictors alone are incomplete, and more factors should be considered for better predictions on the accuracy of cover estimates. For example, shadows that frequently occur in high-spatial resolution imagery might contribute significantly to errors in cover estimates (Fensham et al. 2002; Zhou et al. 2009). Therefore, the application of radiometric enhancement (or restoration) methods for shadow removal may alleviate the shadow problem, and thus improve the accuracy of cover estimates (Zhou et al. 2009).

It is important to note that the accuracy of estimating the cover of features within a patch may be affected by the spatial resolution of imagery. For example, previous studies indicated that the exaggeration of crown cover was scale dependent, decreasing with the increase of scale of photography (Fensham and Fairfax 2002; Fensham et al. 2002). In this study, we only used the $0.6 \mathrm{~m}$ aerial imagery. Therefore, cross-scale (i.e., multiple spatial resolution) evaluation is recommended in future studies. In addition, it should be noted that there are classification errors associated with the reference land cover map. Therefore, some of the "errors" occurring in the visually interpreted classification map were in fact due to errors in the reference map.

\section{Summary and conclusions}

Pattern analysis of landscapes is frequently conducted in an effort to link pattern to ecological processes. As a consequence, the accuracy of that pattern analysis is critical (Wu and Hobbs 2002; Iverson 2007). Knowledge of the magnitude of the errors in pattern analysis, 
and the factors that affect the generation of errors, is needed ( $\mathrm{Li}$ and $\mathrm{Wu}$ 2007; Shao and $\mathrm{Wu} 2008$ ). This research examines the quantitative relationships of whole-patch characteristics and within-patch composition and configuration of land cover features with the accuracy of estimating, by visual interpretation, the cover of those features. The results indicate that, though all factors significantly affected the accuracy of cover estimates, within-patch configuration of the features is the most significant. Most frequently, however, a combination of indicators of whole-patch and within-patch characteristics provides the best prediction of accuracy of cover estimates. In general, errors of cover estimates based on visual interpretation are more likely to occur when patches are smaller or have more complex shapes, and land cover features within a patch are (1) more diverse; (2) more fragmented; (3) more complex in shape; and (4) physically less connected.

Though we used a land cover layer created by a new classification, HERCULES, for this analysis, the approach and results are applicable for any analysis using thematic maps based on visual estimation of land cover features to describe landscape pattern. Our results provide insights into increasing understanding of the link between the accuracy of thematic maps based on visual interpretation and the heterogeneity of land cover features. In addition, the logistic regression models provide a useful, predictive tool for determining under what circumstances estimation error is most likely to occur. This provides an important first step towards a quantitative, spatially explicit model for error prediction of cover estimates based on visual interpretation.

Acknowledgments This research was funded by the National Science Foundation LTER program (grant DEB 042376), and CAREER program (DEB-0844778).

\section{References}

Agresti A (1996) An introduction to categorical data analysis. Wiley, New York

Akaike H (1973) Information theory and an extension of the maximum likelihood principle. In: Second international symposium on information theory. Akademiai Kaidó, Budapest

Allard A, Nilsson B, Pramborg K, Ståhl G, Sundquist S (2003) Manual for aerial photo interpretation in the national inventory of landscapes in Sweden NILS. Department of Forest Resource Management and Geomatics, Umeå
Antrop M, Van Eetvelde V (2000) Holistic aspects of suburban landscapes: visual image interpretation and landscape metrics. Landsc Urban Plan 50:43-58

Blaschke T (2010) Object based image analysis for remote sensing. ISPRS J Photogramm Remote Sens 65(1):2-16

Burnham KP, Anderson DR (2002) Model selection and multimodel inference: a practical information-theoretic approach. Springer, New York

Cadenasso ML, Pickett STA, Schwarz K (2007) Spatial heterogeneity in urban ecosystems: reconceptualizing land cover and a framework for classification. Front Ecol Environ 5:80-88

Cherrill A, McClean C (1995) An investigation of uncertainty in field habitat mapping and the implications for detecting land cover change. Landscape Ecol 10(1):5-21

Clehmann CER, Prior LD, Bowman DMJS (2009) Decadal dynamics of tree cover in an Australian tropical savanna. Austral Ecol 34:601-612

Congalton RG, Mead RA (1983) A quantitative method to test for consistency and correctness in photointerpretation. Photogramm Eng Remote Sens 49(1):69-74

Ellis EC, Wang H (2006) Estimating area errors for fine-scale feature-based ecological mapping. Int $\mathrm{J}$ Remote Sens 27(21):4731-4749

Ellis EC, Wang H, Xiao H, Peng K, Liu XP, Li SC, Ouyang H, Cheng X, Yang LZ (2006) Measuring long-term ecological changes in densely populated landscapes using current and historical high resolution imagery. Remote Sens Environ 100(4):457-473

Fensham RJ, Fairfax RJ (2002) Aerial photography for assessing vegetation change: a review of applications and the relevance of findings for Australian vegetation history. Aust $\mathrm{J}$ Bot 50:415-429

Fensham RJ, Fairfax RJ (2003) Assessing woody vegetation cover change in north-west Australian savanna using aerial photography. Int J Wildl Fire 12(4):359-367

Fensham RJ, Fairfax RJ (2007) Effect of photoscale, interpreter bias and land type on woody crown-cover estimates from aerial photography. Aust J Bot 55:457-463

Fensham RJ, Fairfax RJ, Holman JE, Whitehead PJ (2002) Quantitative assessment of vegetation structural attributes from aerial photography. Int J Remote Sens 23(11):2293-2317

Gill SE, Handley JF, Ennos AR, Pauleit S, Theuray N, Lindley SJ (2008) Characterising the urban environment of UK cities and towns: a template for landscape planning. Landsc Urban Plan 87:210-222

Groom G, Mucher CA, Ihse M, Wrbka T (2006) Remote sensing in landscape ecology: experiences and perspectives in a European context. Landscape Ecol 21:391-408

Gustafson EJ (1998) Quantifying landscape spatial pattern: what is the state of the art? Ecosystems 1:143-156

Heiskanen J, Nilsson B, Mäki AH, Allard A, Moen J, Holm S, Sundquist S, Olsson H (2008) Aerial photo interpretation for change detection of treeline ecotones in the Swedish mountains. Sveriges lantbruksuniversitet, Umeå

Hosmer D, Lemeshow S (2000) Applied logistic regression, 2nd edn. Wiley, New York

Iverson L (2007) Adequate data of known accuracy are critical to advancing the field of landscape ecology. In: Wu J, Hobbs R (eds) Key topics in landscape ecology. Cambridge University Press, Cambridge, pp 11-38 
Li H, Wu J (2007) Landscape pattern analysis: key issues and challenges. In: Wu J, Hobbs R (eds) Key topics in landscape ecology. Cambridge University Press, Cambridge, pp 39-61

Lillesand TM, Kiefer RW (2004) Remote sensing and image interpretation. Wiley, New York

McGarigal K, Cushman SA, Neel MC, Ene E (2002) FRAGSTATS: spatial pattern analysis program for categorical maps. Computer software program. University of Massachusetts, Amherst. http://www.umass.edu/landeco/research/ fragstats/fragstats.html. Accessed 6 April 2012

Nagelkerke NJD (1991) A note on a general definition of the coefficient of determination. Biometrika 78(3):691-692

Paine DP, Kiser JD (2003) Aerial photography and image interpretation, 2nd edn. Wiley, Hoboken

Richards JA, Jia X (2006) Remote sensing digital image analysis. Springer, New York

Schumaker NH (1996) Using landscape indices to predict habitat connectivity. Ecology 77:1210-1225

Shao G, Wu J (2008) On the accuracy of landscape pattern analysis using remote sensing data. Landscape Ecol 23:505-511

Shao G, Liu D, Zhao G (2001) Relationships of image classification accuracy and variation of landscape statistics. Can J Remote Sens 27(1):33-43

Smith JH, Wickham JD, Stehman SV, Yang LM (2002) Impacts of patch size and land-cover heterogeneity on thematic image classification accuracy. Photogramm Eng Remote Sens 68:65-70

Smith JH, Stehman SV, Wickham JD, Yang LM (2003) Effects of landscape characteristics on land-cover class accuracy. Remote Sens Environ 84:342-349
Ståhl G, Allard A, Esseen P, Glimskär A, Ringvall A, Svensson J, Sundquist S, Christensen P, Torell A, Högström M, Lagerqvist K, Marklund L, Nilsson B, Inghe O (2011) National Inventory of Landscapes in Sweden (NILS) scope, design, and experiences from establishing a multiscale biodiversity monitoring system. Environ Monit Assess 173:579-595

Turner MG (2005) Landscape ecology: what is the state of the science? Annu Rev Ecol Evolution Syst 36:319-344

Turner MG, Gardner RH, O’Neill RV (2001) Landscape ecology in theory and practice: pattern and process. Springer, New York

Wagenmakers EJ, Farrell S (2004) AIC model selection using Akaike weights. Psychon Bull Rev 11(1):192-196

Wu J, Hobbs R (2002) Key issues and research priorities in landscape ecology. Landscape Ecol 17:355-365

Zhou W, Troy A (2008) An object-oriented approach for analyzing and characterizing urban landscape at the parcel level. Int J Remote Sens 29(11):3119-3135

Zhou W, Huang G, Troy A, Cadenasso ML (2009) Object-based land cover classification of shaded areas in high spatial resolution imagery of urban areas: a comparison study. Remote Sens Environ 113(8):1769-1777

Zhou W, Schwarz K, Cadenasso ML (2010) Mapping urban landscape heterogeneity: agreement between visual interpretation and digital classification approaches. Landscape Ecol 25(1):53-67 